\title{
TV/Series
}

Hors séries 1 | 2016

Lost: (re)garder l'île

\section{La fin de Lost, le paradoxe des séries et l'expérience de la perte}

\section{Hugo Clémot}

\section{(2) OpenEdition}

1 Journals

Édition électronique

URL : http://journals.openedition.org/tvseries/1710

DOI : 10.4000/tvseries. 1710

ISSN : 2266-0909

Éditeur

GRIC - Groupe de recherche Identités et Cultures

Référence électronique

Hugo Clémot, «La fin de Lost, le paradoxe des séries et l'expérience de la perte », TV/Series [En ligne], Hors séries 1 | 2016, mis en ligne le 01 octobre 2016, consulté le 02 mai 2019. URL : http:// journals.openedition.org/tvseries/1710; DOI : 10.4000/tvseries.1710

Ce document a été généré automatiquement le 2 mai 2019.

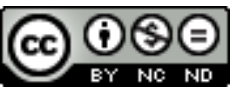

TV/Series est mis à disposition selon les termes de la licence Creative Commons Attribution - Pas d'Utilisation Commerciale - Pas de Modification 4.0 International. 


\title{
La fin de Lost, le paradoxe des séries et l'expérience de la perte
}

\author{
Hugo Clémot
}

1 La fin de Lost a suscité l'une des plus grandes déceptions de l'histoire des séries télévisées, au point de constituer une référence quand on parle d'une série dont la fin est décevante ${ }^{1}$. La question de savoir pourquoi tant de spectateurs furent si déçus par cette fin pose le problème conceptuel de la clôture narrative, c'est-à-dire la difficulté de savoir expliquer ce qui peut provoquer l'impression de finalité que le spectateur s'attend à éprouver devant la plupart des œuvres audiovisuelles. Dans ce qui suit, je m'appuie sur Lost pour présenter ce problème dont on débat en philosophie contemporaine du cinéma depuis que le philosophe américain Noël Carroll a proposé de le résoudre au moyen d'un modèle "érotétique ». Convaincu de l'insuffisance de cette théorie, je lui oppose un modèle « téléologique » qui soulève un autre problème que je propose d'appeler le "paradoxe des séries » et dont la résolution exige à mon sens que l'on adopte une autre approche des séries télévisées, ce qui permet en outre de reconnaître que la fin de Lost est d'un certain point de vue réussie.

\section{Le problème de la clôture narrative : modèles érotétique et téléologique}

\section{1) L'analyse carrollienne de la clôture narrative}

Dans une série d'articles commencée en $1984^{2}$, le philosophe analytique Noël Carroll a proposé un modèle "érotétique » du récit pour rendre compte de la « clôture narrative » (narrative closure) cinématographique. Cette conception soutient que l'impression de finalité ${ }^{3}$ ou de résolution que l'on ressent à la fin de la plupart des films est provoquée par le fait que les questions que le film a soulevées dans l'esprit du spectateur trouvent une réponse. Ce besoin de réponse à nos questions et le plaisir que nous en retirons, et que nous retirons donc des récits, tiendraient à la nature humaine. 
Partant d'une conception causaliste du récit selon laquelle un discours est un récit si et seulement s'il décrit au moins deux événements temporellement et causalement reliés l'un à l'autre ${ }^{4}$, Carroll introduit le modèle érotétique en vue de répondre à l'objection suivante : si le récit compte, au titre de ses éléments nécessaires, une connexion narrative 5, c'est-à-dire une relation causale entre événements, comment rendre compte de l'impression de clôture que l'on ressent quand toutes les questions posées par le récit ont trouvé une réponse, puisque le seul fait d'observer une suite de relations causales ne semble pas suffisant pour nous émouvoir? Quelle est l'origine de ce sentiment? Selon le modèle érotétique de la relation interrogative utilisé par Poudovkine ${ }^{6}$, le sentiment de clôture tiendrait à la satisfaction d'un besoin naturel de trouver des réponses à nos questions. Les narrateurs s'appuieraient sur ce besoin pour relater les événements d'une telle façon que le spectateur se pose plus ou moins consciemment certaines questions et obtienne ensuite des réponses ${ }^{7}$, quitte à manipuler la temporalité 8 . L'usage du flashback ou du flashforward est évidemment l'un des principes formels importants de Lost ${ }^{9}$. Par exemple, dans l'épisode centré sur Sawyer (S1E8), les spectateurs sont invités à se demander pourquoi le personnage est si méchant, interrogation à laquelle les flashbacks permettent de répondre : c'est parce qu'il a été lui-même victime d'un méchant - un escroc qu'il a imité au point de prendre son identité. Le modèle érotétique permet aussi de comprendre comment des épisodes situés dans des saisons différentes peuvent fonctionner ensemble pour maintenir l'attention des spectateurs. Ainsi l'épisode S2E10 ("The 23rd Psalm») vise-t-il à répondre à la question soulevée dans l'épisode S1E19 ("Deus ex machina »): que faisaient des trafiquants de drogue nigériens, déguisés en prêtres, dans le petit avion qui s'est écrasé sur l'île? Ce modèle permet même de récompenser l'attention des spectateurs les plus perspicaces : dans l'épisode S2E15, une réponse est donnée à la question de savoir pourquoi Sawyer porte de nouvelles lunettes, question qui avait, certes, été préparée deux épisodes plus tôt quand Sawyer expliquait à Kate qu'il avait perdu ses anciennes lunettes sur le radeau.

Figure 1 : Sawyer dans S2E13

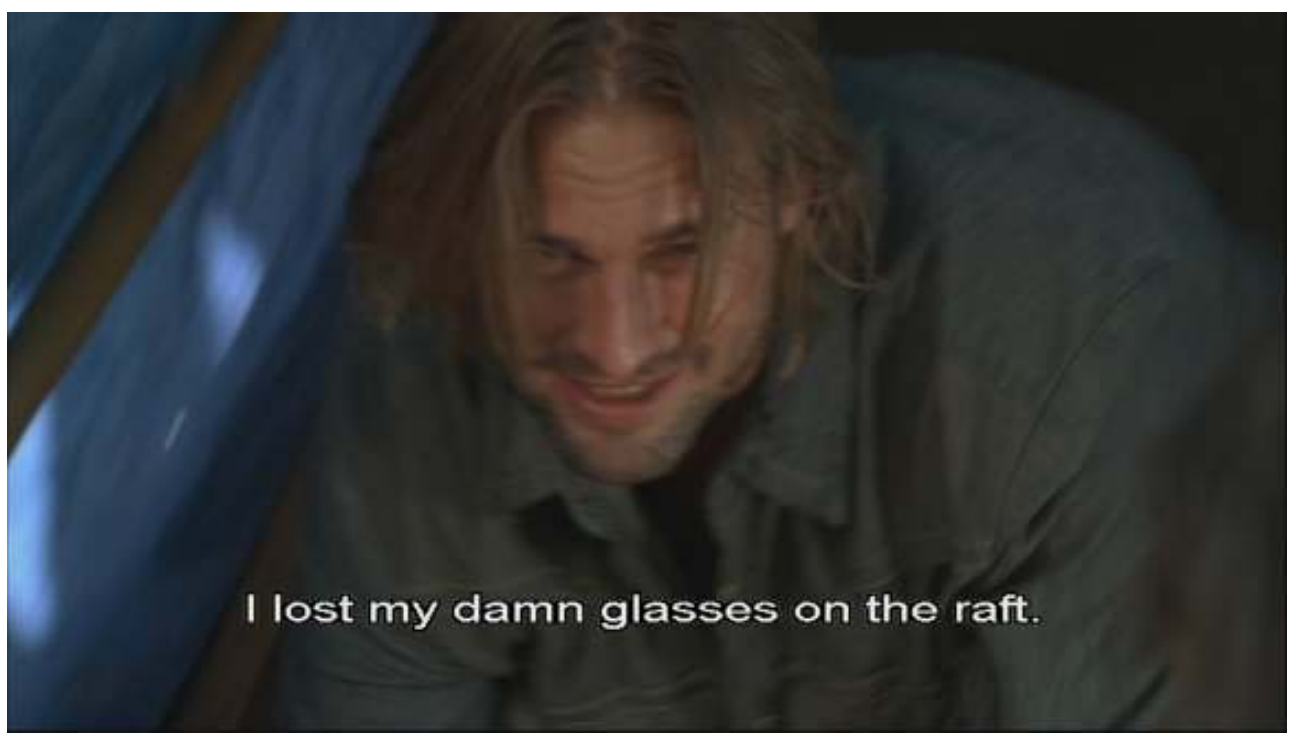




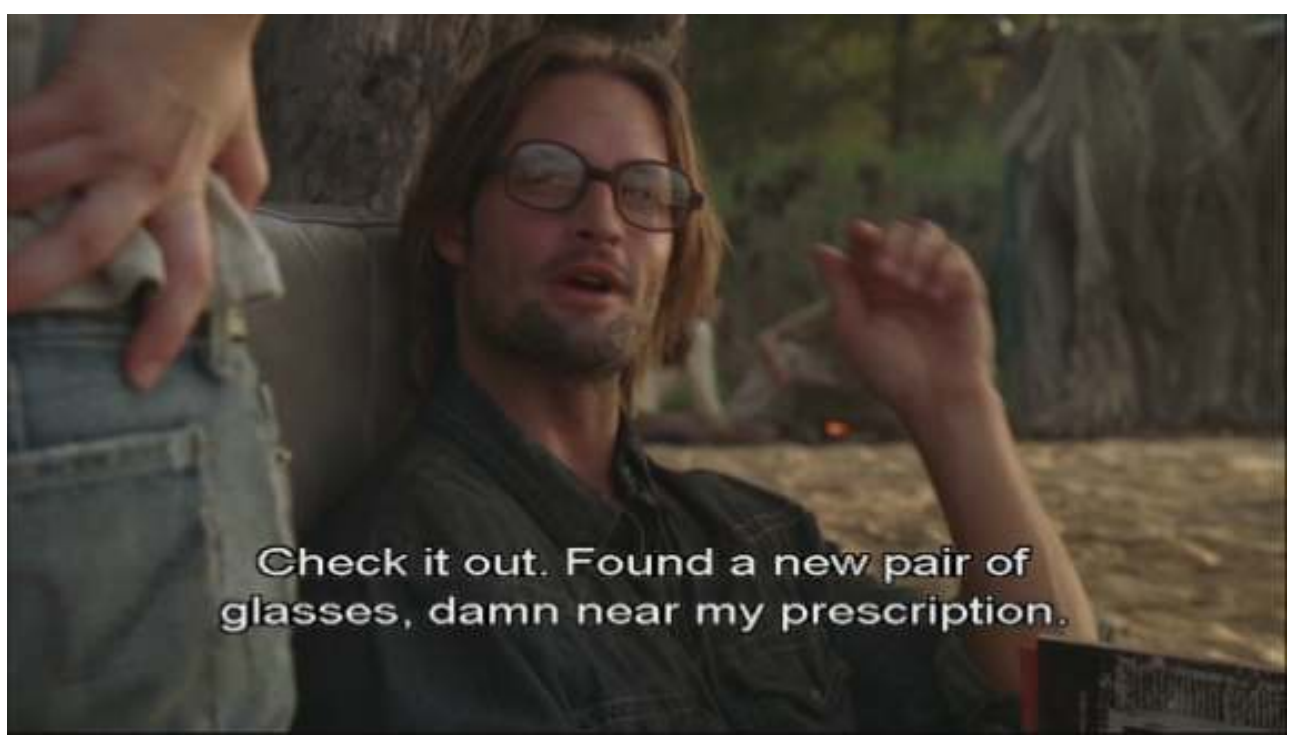

Pour illustrer cette définition de la clôture narrative, Carroll donne un contre-exemple : dans le film Les Berkman se séparent (The Squid and the Whale, 2005) de Noah Baumbach, le réalisateur insiste à plusieurs reprises sur la tendance alcoolique de l'un des personnages, le frère cadet, Frank, sans que la question de savoir ce qui va lui arriver, et notamment quelles conséquences son "problème" va avoir pour l'histoire, ne trouve de réponse. Carroll fait remarquer que, dans certains cas, cette absence de clôture peut provoquer l'agacement du spectateur, agacement qui viendrait de ce que la promesse implicite faite au spectateur de lui fournir toutes les réponses aux questions qu'il s'est posées n'a pas été tenue ${ }^{10}$. Cela semble correspondre à l'expérience des spectateurs à la fin de Lost, expérience verbalisée très tôt par le personnage qui représente souvent la position des spectateurs, Hugo Reyes dit "Hurley ». Dans l'épisode S1E18 (" Numbers »), il rappelle certaines des questions laissées sans réponse depuis le début de la série, avant d'exiger violemment de Danielle Rousseau qu'elle lui explique ce que signifie la suite de nombres qui rend fou ${ }^{11}$.

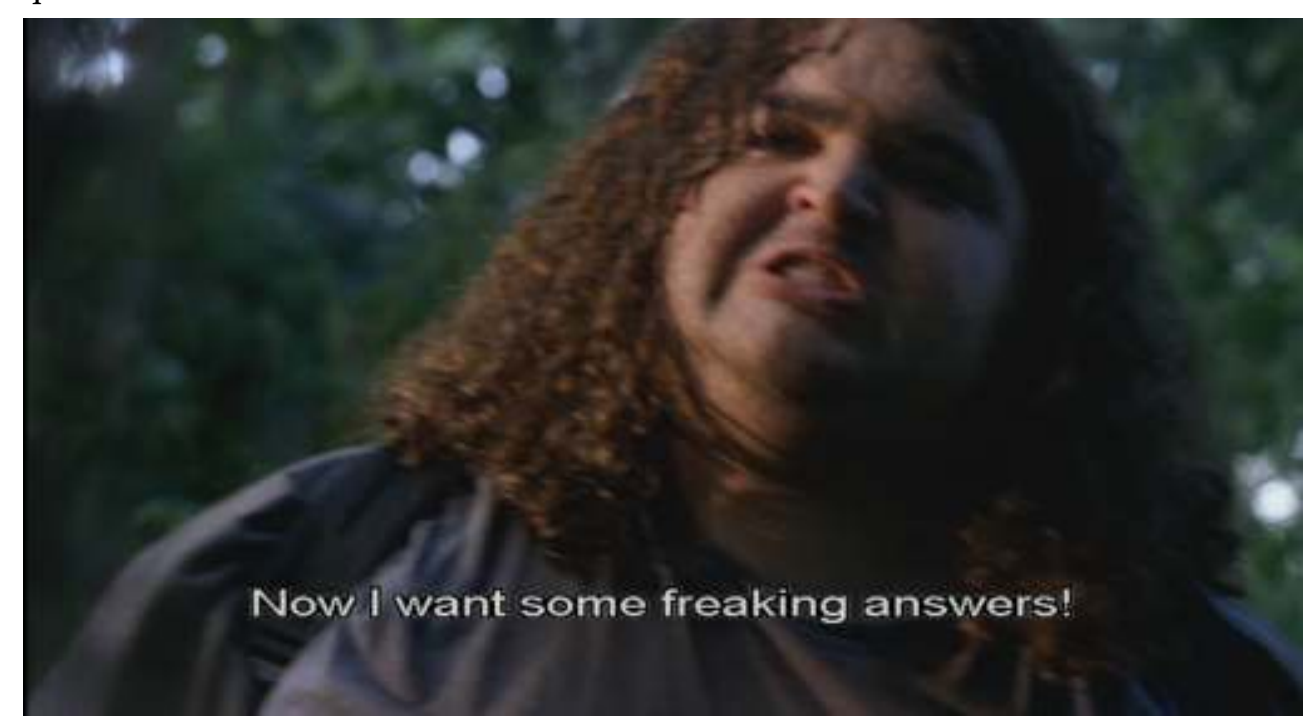


Figure 3 : Hurley dans S1E1 8

5

Carroll soutient donc que la finalité n'est pas présente dans le récit: elle serait une impression qui naîtrait quand la tension mentale liée à la recherche de réponses aux questions soulevées par le récit se relâche, tension elle-même née d'un besoin naturel de réponse à nos questions. À suivre cette théorie, la série Lost aurait déçu car elle n'aurait pas su répondre à toutes les questions qu'elle avait soulevées dans l'esprit des spectateurs: la promesse, implicite ou explicite, de résolution n'aurait pas été tenue, l'attention du spectateur n'aurait pas été payée en retour et sa déception aurait été légitime.

\section{2) La clôture narrative n'est-elle qu'une impression ?}

On peut critiquer un présupposé commun à Carroll et à d'autres philosophes analytiques du cinéma comme David Velleman ${ }^{12}$. En effet, l'un et l'autre s'accordent à dire que la clôture narrative est une émotion qui naît de la satisfaction d'un besoin naturel, qu'il s'agisse de trouver des réponses à nos questions ou de relâcher la pression émotionnelle exercée par le début d'une histoire. On trouve, par exemple, une référence directe à cette théorie naturaliste dans l'épisode S1E8 de la série Dollhouse (Joss Whedon, 2009-2010), lorsque le Dr Saunders/Whiskey explique que «[1]e cerveau de chaque agent a été programmé pour libérer un sédatif quand il ressent une résolution ${ }^{13} »$. Or, suffit-il de mentionner un besoin naturel pour expliquer l'impression de finalité ? En effet, si l'on peut comprendre que l'esprit exige certaines réponses, on comprend moins qu'il exige des réponses à toutes les questions, par exemple aussi bien «Que veut dire "Rosebud" dans Citizen Kane (réal. Orson Welles, 1941) ?» ou « Que veut dire la suite 4814152342 dans Lost ?» que « Pourquoi la panthère est-elle rose dans The Pink Panther (réal. Blake Edwards, 1963-) ${ }^{14}$ ? » ou « Pourquoi n'y a-t-il pas plus de serpents sur l'île dans Lost? » ou encore « Pourquoi y avait-il autant de bâches bleues dans l'avion de Lost? », et l'on ne comprend plus pourquoi Carroll peut être agacé parce qu'un film ne lui dit pas ce qu'il advient d'un personnage secondaire prépubère aux tendances alcooliques ${ }^{15}$, alors qu'il n'est d'habitude pas frustré de ne pas savoir exactement combien d'enfants eurent les héros (dont on nous dit à la fin qu'ils vécurent heureux). C'est comme si l'on disait qu'il est agaçant de ne pas savoir ce qui est arrivé à tel personnage blond présent à l'enterrement de Boone en S1E21, mais qu'il n'est pas agaçant ne pas savoir ce que font tout le temps tous les personnages secondaires. 
Figures $4 a$ et $4 b$ : Le groupe de survivants dans S1E21
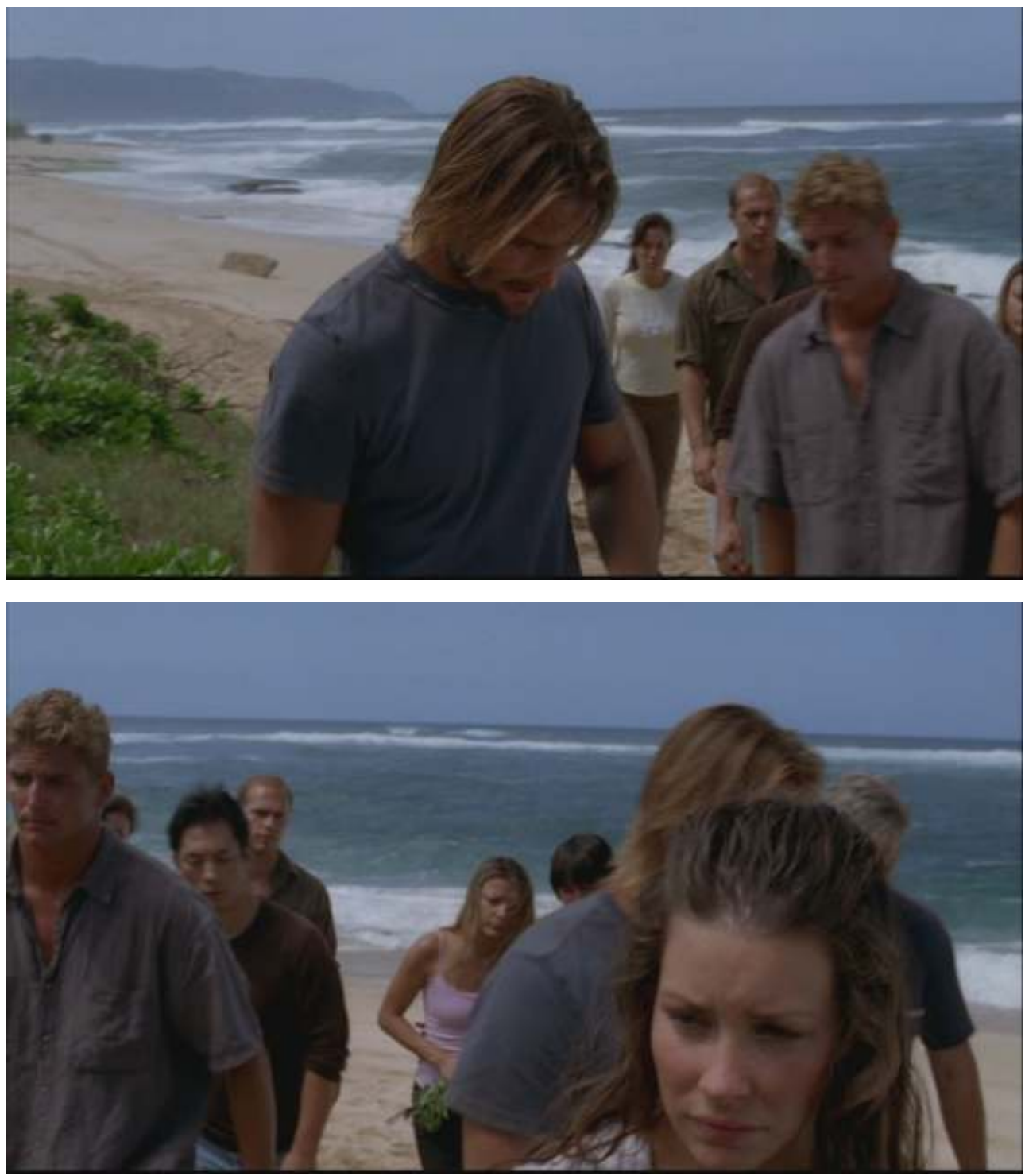

7 L'idée n'est pas qu'il faudrait s'en agacer, puisque ce serait absurde et idiot, aussi idiot que de gesticuler quand on manipule un bâton de dynamite, mais plutôt qu'il doit y avoir quelque chose qui permet au spectateur de savoir comment se poser les bonnes questions. Carroll prétend rendre compte du phénomène de l'agacement ressenti lorsqu'une question posée par un récit ne trouve pas de réponse en parlant d'une "promesse implicite ${ }^{16}$ » faite par le réalisateur de récompenser notre attention, en fournissant toutes les informations nécessaires pour répondre aux questions que le film pourrait susciter en nous.

8 Mais puisque chaque image d'un film pourrait susciter un nombre indéfini de questions dans l'esprit de chaque spectateur, cette explication ne semble pas suffire. Par exemple, dans la scène où les rescapés du crash aérien s'accordent pour confier la garde de l'arme à feu à Kate ${ }^{17}$ (S1E3), le gros plan d'un revolver pourrait susciter différentes questions chez différents spectateurs, qui ne trouveraient pas de réponse par la suite ("Quelle est la marque de ce revolver?", "Où a-t-il été acheté ?", «Par qui a-t-il été fabriqué ?», «Pourquoi a-t-il cette couleur? », «A-t-il été bien nettoyé ?», etc.) Autrement dit, l'idée 
de clôture narrative ne saurait s'expliquer seulement par un besoin naturel de réponse à nos questions dans la mesure où il importe surtout de savoir quelles questions les récits nous font nous poser et quel type de réponses peut nous satisfaire. Pour éviter la conclusion absurde selon laquelle l'impression de résolution se produirait toujours par hasard, c'est-à-dire quand l'esprit du spectateur parvient spontanément à s'arrêter sur les bonnes questions, il faut compléter le modèle en précisant un critère d'identité de ces questions pertinentes.

On peut soutenir que s'il y a bien une expérience qui correspond au suivi de la dernière phase du récit, de sa fin et qui peut être envisagée comme étant provoquée par l'obtention d'une réponse, il ne s'agit que d'un symptôme et non d'un critère de la clôture narrative elle-même. En effet, on peut penser que le critère qui nous permet spontanément d'identifier quelles sont les questions importantes pour suivre le récit est téléologique : une question méritera d'être posée si elle porte sur une action que l'on peut tenir pour la réalisation d'un but, d'une fin (en grec, telos) ou d'un moyen d'atteindre ce but qui a animé l'un des personnages principaux. Par exemple, une explication téléologique générale de Lost pourrait prendre la forme suivante : pour sauver le monde, il faut protéger le cœur de l'île ; pour protéger le cœur de l'île, il faut un gardien ; pour qu'il y ait un bon gardien, il faut qu'il ait le bon profil, qu'il connaisse et soit attaché à l'île et qu'il passe un certain nombre d'épreuves; pour qu'il ait le bon profil, il faut étudier sa biographie; pour qu'il connaisse et soit attaché à l'île et qu'il passe un certain nombre d'épreuves, il faut le faire venir sur l'île ; pour qu'il vienne sur lîle, il faut que son avion s'écrase sur l'île ; etc.

On remarquera que cette explication téléologique n'est rien d'autre que le raisonnement pratique de Jacob, tel qu'on peut le reconstruire après avoir vu la série: comme dans l'analyse d'Aristote ${ }^{18}$, la prémisse initiale introduit un but, une fin de l'acteur, tandis que les prémisses suivantes présentent différents moyens d'atteindre cette fin, éventuellement reliés par des relations téléologiques de moyen à fin, jusqu'à atteindre une conclusion qui est l'action concrète à effectuer. Dans cette perspective, la clôture narrative n'est pas extérieure à l'histoire en tant qu'elle serait une impression psychologique du spectateur, mais un moment essentiel du récit auquel les autres sont subordonnés.

\section{3) Modèles érotétique et téléologique de l'impression de clôture narrative}

11 La force de l'intuition de Carroll relative au modèle érotétique est d'avoir montré que la structure des histoires pouvait souvent être découverte en cherchant quelles questions et quelles réponses étaient soulevées ou données au cours du récit ${ }^{19}$. Son importance demeure, même s'il faut la compléter en tenant compte du rôle joué par les pouvoirs causaux et la téléologie dans notre conception du récit. En effet, les questions que les récits soulèvent sont souvent l'énoncé, sous forme interrogative, des pouvoirs naturels et intentionnels des agents, c'est-à-dire des tendances que l'on attribue aux agents. Ainsi lorsqu'un homme et une femme célibataires se rencontrent pour la première fois, tels Jack et Kate, nous nous demandons s'ils pourront tomber amoureux. Quand dans l'épisode S01E24 Aaron, le fils de Claire, se fait kidnapper, nous nous demandons s'il pourra retrouver sa liberté. Dans les épisodes S1E24-25, connaissant le pouvoir explosif de la nitroglycérine et le pouvoir combustible de la mèche reliée au bâton de dynamite, nous 
pouvons anticiper le cours des événements, ce qui nous invite à nous poser la question, typiquement érotétique : l'explosion de la dynamite sera-t-elle empêchée par Hurley, qui cherche à éteindre la mèche, ou bien aura-t-elle lieu et ouvrira-t-elle un accès à la trappe?

Ce qui nous permet de poser cette question et d'appliquer le modèle érotétique à l'analyse $\mathrm{du}$ récit filmique, c'est bien l'analyse téléologique que nous faisons plus ou moins spontanément des événements : c'est parce que nous connaissons les pouvoirs et les fins naturels ou intentionnels des agents que nous ne sommes pas étonnés par leurs comportements ou que, le cas échéant, nous réussissons à dépasser cet étonnement pour l'intégrer dans une structure téléologique plus large que celle dont nous sommes initialement partis. Nos attentes reposent sur «notre sens de ce qui est possible, étant donnés les événements antérieurs ${ }^{20} "$, comme l'écrit Carroll, mais ce sens relève plus généralement de la capacité à raisonner pratiquement ou causalement dont doivent faire preuve, par exemple, le juge, le médecin, mais aussi le garagiste.

conception est correcte, alors nous pouvons rendre compte du désir de clôture narrative ainsi : si nous acceptons d'entendre le détail des événements qui ont conduit au résultat finalement obtenu et d'attendre ainsi que l'on nous dise la fin, c'est parce que nous désirons comprendre. Or, on comprend quand on peut mentionner l'action d'un agent, c'est-à-dire quels moyens un agent a adoptés pour atteindre sa fin. Autrement dit, on comprend quand on pourrait produire, à la demande, un raisonnement causal ou pratique susceptible de décrire le cours des événements en mentionnant les moyens nécessaires et conjointement suffisants pour produire le résultat espéré.

Que le modèle érotétique ne soit qu'un cas particulier de l'explication téléologique semble donc prouver que l'on rend mieux compte de l'impression de résolution quand on la définit comme le sentiment qui accompagne un récit dont l'apparence est finalisée, plutôt que comme l'émotion provoquée par le fait que toutes nos questions trouvent une réponse. En effet, il est possible que certaines questions demeurent sans réponse et que nous ayons pourtant l'impression d'une finalité à condition que cette absence de réponses ne nous empêche pas de reconstruire les raisonnements pratiques des agents. Il nous est en effet permis dans ce cas de comprendre les actions des agents comme autant de moyens d'atteindre leurs fins. Il se pourrait donc que nos questions ne trouvent pas toutes de réponse sans que nous cessions d'éprouver un sentiment de finalité, mais nous ne pourrions avoir cette impression s'il n'était pas possible de hiérarchiser de façon téléologique au moins certaines des actions qui nous sont décrites. Dans cette perspective, on peut soutenir que la série est réussie ${ }^{21}$ si l'on accepte de croire en la finalité du récit. Il n'est cependant pas sûr qu'il soit possible de déterminer aussi abstraitement les raisons du plaisir que nous prenons à voir un film ou une série se terminer, sans partir de l'expérience concrète d'une œuvre particulière. Or, dès qu'on s'intéresse à ce que la série Lost a à dire sur la question de la téléologie, on s'aperçoit qu'elle tient un discours particulièrement pertinent et qui témoigne d'une conscience aiguë des enjeux et de la complexité du problème.

En effet, l'opposition centrale entre Jack Shephard et John Locke est aussi, comme l'a montré Pacome Thiellement ${ }^{22}$, une opposition entre deux types de spectateurs de Lost. Comme le dit Locke dans la deuxième partie de l'épisode S1E25, Jack et lui « ne voient pas les choses de la même manière " ("you and I don't see eye-to-eye sometimes ») : Jack est un " homme de science » selon Locke, convaincu que les événements arrivent par le jeu du hasard et de la nécessité de la lutte pour la survie, sans qu'il y ait forcément d'espoir 
d'atteindre une clôture, tandis que Locke est un homme de foi, convaincu qu'il y a une raison (purpose) qui expliquerait pourquoi l'avion s'est écrasé, pourquoi ils ont survécu, etc. Il est tentant, à l'instar de Pacome Thiellement dans son intervention au Forum des images, de lire cette séquence comme une mise en demeure du spectateur de Lost. Ce dernier serait contraint de choisir à l'intérieur d'une stricte alternative: "soit tout accepter, soit tout refuser [...] Accepter ou ne pas accepter de faire un saut dans la foi [...] [C]roire à une dimension mythique de l'existence ou à un pur réalisme désenchanté23. " Concernant la clôture narrative, il est également tentant de doubler cette opposition entre raison et foi, science et religion, absurde et sens, d'une opposition entre conceptions érotétique (naturaliste) et téléologique (finaliste) de la clôture.

Dans Lost, il n'y a pas qu'Hurley qui soutienne cette conception naturaliste du sentiment de finalité ; le docteur Jack Shephard adhère aussi à cette perspective quand il s'adresse à Juliet (S03E07, « Not in Portland ») : « Après tout ce que j'ai traversé, vous me devez une

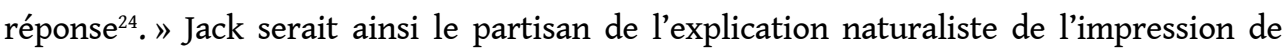
finalité, tandis que Locke défendrait l'idée que l'impression correcte dérive de la perception d'une authentique finalité. Cependant, le renversement répété des perspectives - Locke perd la foi quand Jack se convertit à son point de vue - conduit à supposer que la série est bien davantage habitée par le scepticisme moderne, c'est-à-dire par le problème d'avoir à choisir en des temps d'urgence et d'incertitude.

\section{S'orienter quand on est perdu : Lost et l'expérience de la perte}

\section{1) La figure du voyageur perdu dans la forêt et le scepticisme du personnage, de l'homme contemporain et du spectateur}

17 La figure du voyageur perdu dans la forêt que l'on retrouve en S1E11 est, au moins depuis Descartes, la figure de l'individu confronté au scepticisme pratique, c'est-à-dire contraint ("nous sommes embarqués ", écrivait Pascal) de faire des choix dans l'urgence et sans savoir avec certitude ce qui est bon ${ }^{25}$. On la retrouve, par exemple, encore aujourd'hui, dans les nombreux films et séries télévisées de zombies où le héros, fuyant les villes infestées, cherche refuge dans ces zones réputées inhabitées. On peut y voir une "paraphrase» artistique de notre scepticisme contemporain, c'est-à-dire de notre difficulté à comprendre un monde plein de dangers et de catastrophes et à savoir comment y agir en se protégeant des menaces ${ }^{26}$. Les multiples références littéraires, cinématographiques et télévisuelles sont aussi là pour montrer ce que les études de sociologie de la réception ne font que confirmer: les spectateurs de récits de fiction trouvent dans ces œuvres des moyens de penser leur existence et leurs difficultés ordinaires. Par exemple, dans l'épisode S5E13, Hurley analyse la situation de Miles vis-àvis de son père, Pierre Chang, en mobilisant l'exemple fictionnel de la relation de Luke à Dark Vador dans Star Wars (réal. George Lucas, 1977), affirmant la fonction épiphanique de cette œuvre de la culture populaire. Le projet qu'a Hurley d'écrire, en l'améliorant dès 1977, le scénario de L'Empire contre-attaque (1980) et de l'envoyer à George Lucas n'est donc pas futile. La paraphrase de notre scepticisme est présente dans Lost ne serait-ce que dans le commentaire que livre Kate à Juliet dans l'épisode S3E15, lorsqu'elles sont menottées l'une à l'autre dans la forêt : « Bienvenue dans le monde merveilleux où l'on ne comprend pas ce qui se passe ${ }^{27}$.» 

réflexif de la série parmi d'autres, qui montre que le scepticisme, dans Lost, n'est pas seulement présent chez les personnages ${ }^{28}$, ni conçu pour nous permettre de penser notre situation dans le monde. Il est aussi là, de manière peut-être plus intéressante, pour aider à domestiquer et à contenir le scepticisme de spectateurs qui ne croient plus aussi facilement aux histoires qu'on leur raconte. Après le cinéma d'auteur, le cinéma populaire est entré à son tour, au moins depuis le début des années 1990, dans « l'ère du soupçon " moderniste, c'est-à-dire ce moment de l'histoire d'un art où les artistes ne peuvent plus s'appuyer sur les conventions et les genres hérités de la tradition, dans la mesure où les spectateurs eux-mêmes ont cessé d'y croire. La série Lost serait une tentative moderniste pour surmonter cette difficulté en affrontant le scepticisme des spectateurs.

Encouragé par le modèle téléologique de la compréhension, le scepticisme du spectateur tend à s'exprimer dans les termes suivants : soit il est possible de connaître les fins des personnages et de situer chaque événement narratif dans une explication téléologique, soit le récit n'a aucun sens. Mais comme il est impossible à un récit d'être entièrement cohérent et qu'il est en revanche toujours possible de considérer que nous ne faisons que projeter sur les personnages et le monde diégétique des fins qui ne s'y trouvent pas, peuton alors croire au récit?

Il est remarquable que ces réflexions soient à peu près celles de Locke, c'est-à-dire du personnage qui est à la fois le plus dogmatique et celui qui traverse les crises sceptiques aux conséquences les plus graves, lorsqu'il découvre en S2E21, dans la station Dharma de La Perle, qu'appuyer sur le bouton dans la trappe n'aurait été qu'une expérience filmée et observée par des scientifiques. Eko essaye alors de lui redonner foi en l'île en lui disant que s'ils appuient sur le bouton, ce n'est pas parce qu'on leur a dit de le faire dans un film, mais parce qu'ils croient qu'ils étaient destinés à le faire. La séquence attire doublement l'attention du spectateur sur sa position vis-à-vis des personnages en lesquels il doit croire pour aimer la série. En effet, est-il bien certain que l'action des personnages ait un sens? Le travail des écrivains ne consisterait-il pas plutôt en une répétition en variation dépourvue de sens, des mêmes faits et gestes des mêmes personnages, un processus qui serait ainsi analogue à celui de la remise à zéro du compteur dans la trappe ${ }^{29}$ ?

\section{2) Le « paradoxe des séries » et l'expérience esthétique}

21 En tant qu'il soutient que l'on comprend le sens d'un récit quand on parvient à concevoir les actions des agents comme des moyens de composer avec les circonstances pour atteindre leurs fins, le modèle téléologique semble présupposer l'existence d'un ordre stable et immuable de relations téléologiques, c'est-à-dire une hiérarchie de fins, qui nous permettraient d'identifier aisément les fonctions des différentes scènes et séquences, qu'on les rapporte, d'un point de vue diégétique, aux buts des personnages ou, d'un point de vue fictionnel, aux intentions des créateurs. D'un point de vue fictionnel, ce modèle semble en effet parfaitement s'accorder avec l'idée de genre cinématographique et donner un contenu à la notion de «lois du genre »: chaque genre cinématographique aurait un ensemble de fins (faire rire, pleurer, etc.) qui détermineraient plus ou moins rigoureusement les moyens de les atteindre, c'est-à-dire les manières de faire ou encore les règles ou lois du genre en question.

TV/Series, Hors séries 1 | 2016 
Cependant, on a découvert, au XX siècle, un nouveau paradoxe sceptique qui met en évidence le fait qu'aucune fin ne peut déterminer rigoureusement la règle pour l'atteindre. Dans son ouvrage Règles et langage privé. Introduction au paradoxe de Wittgenstein ${ }^{30}$, Saül Kripke prétend en effet avoir découvert l'existence d'un "paradoxe sceptique " qui « pose à la philosophie le problème sceptique le plus radical et le plus original qu'elle ait jamais eu à affronter ${ }^{31} \%$. Ce paradoxe serait explicitement énoncé au paragraphe 201 des Recherches philosophiques quand Wittgenstein écrit : « Notre paradoxe était celui-ci : Une règle ne pourrait déterminer aucune manière d'agir, étant donné que toute manière d'agir peut être mise en accord avec la règle ${ }^{32}$. » $\mathrm{Ce}$ " paradoxe des règles " est suffisamment connu pour être expliqué par un personnage à un autre dans un film populaire assez récent: The Oxford Murders (Crimes à Oxford, réal. Alex de la Iglesia, 2008). La présentation qui en est faite dans ce film est remarquable à deux égards pour notre enquête. D'une part, il est dit de ce paradoxe qu'il aurait le pouvoir de rendre fou. On remarque d'ailleurs que cette séquence de Crimes à Oxford se termine sur un plan qui rappelle un autre plan d'un grand film classique sur la maladie mentale, Shock Corridor (réal. Samuel Fuller, 1963), dans lequel un journaliste qui s'est fait passer pour fou afin de mener son enquête finit par le devenir réellement au cours d'une crise qui se passe, elle aussi, dans le couloir d'une institution psychiatrique, alors qu'il est assis à côté d'un individu dont la corpulence rappelle celle d'Hugo Reyes.

Figure 5 : Shock Corridor (réal. Samuel Fuller, 1963)

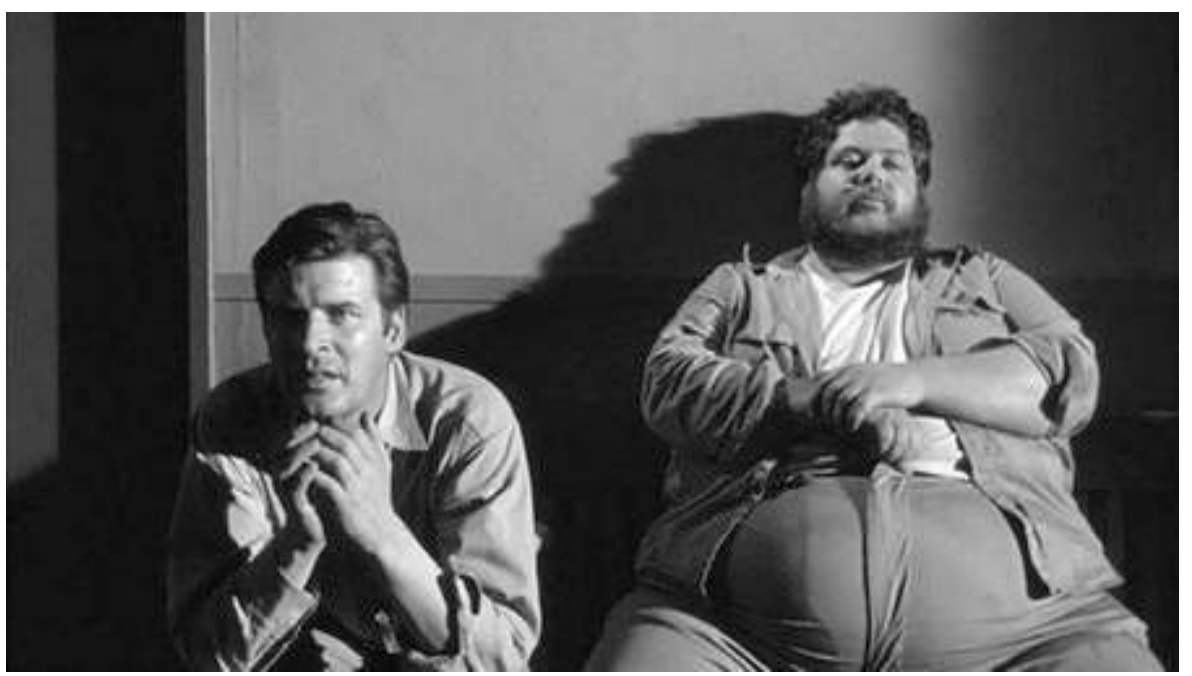


Figure 6 : Hurley sans Dave dans Lost S2E18

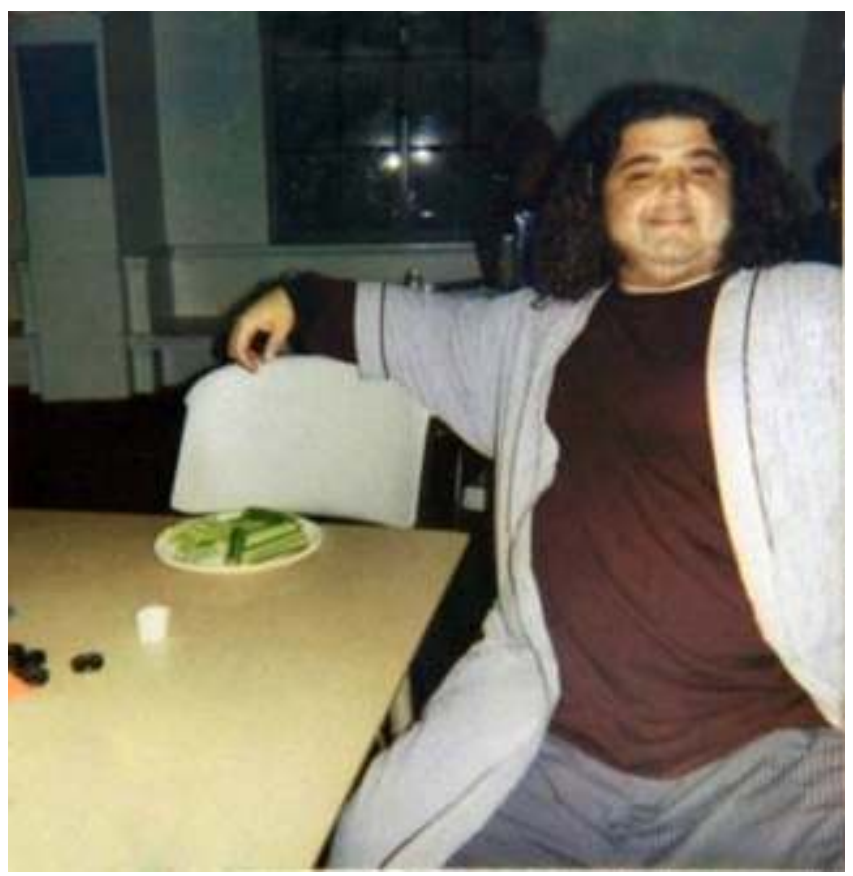

D'autre part, ce paradoxe peut être paraphrasé en mobilisant un exemple - celui de l'activité qui consiste à continuer une suite ou encore une série de nombres pour en comprendre le sens: on pense bien sûr aux nombres $4 \quad 8 \quad 15 \quad 16 \quad 23 \quad 42$. Il semble logiquement toujours possible de poursuivre une série autrement et de modifier ainsi rétrospectivement le sens des nombres ${ }^{33}$ qui la constituent. Mais si l'on se demande si ce paradoxe pourrait valoir non seulement pour les séries numériques, mais aussi pour les séries télévisées, en tant qu'elles sont composées d'une suite d'événements dont le sens semble devoir être déterminé à la fois par les événements passés et par les événements futurs, on s'aperçoit que la réponse est positive : il est toujours possible de poursuivre une série télévisée autrement et de modifier ainsi rétrospectivement le sens des événements passés qui la constituent, ce que la technique des flashsideways de Lost montre relativement bien. Je propose de donner le nom de "paradoxe des séries » à cette idée, dont les conséquences sont, à mon sens, importantes pour la question de la clôture narrative.

En effet, le modèle téléologique présuppose que l'on comprend le sens d'un récit si l'on parvient à faire des événements des moyens pour atteindre les fins des personnages. Mais s'il est toujours possible de poursuivre une série télévisée autrement, alors cela veut dire que l'on ne peut jamais être sûr de connaître ces fins et donc, selon ce même modèle téléologique, que l'on ne peut jamais être sûr de comprendre ce qui nous est raconté. Mais s'il y a là une vérité sceptique, alors cette vérité menace aussi la compréhension que les spectateurs ont de leur histoire personnelle. En effet, les flashbacks par lesquels nous découvrons la vie de chaque survivant peuvent être lus comme des commentaires formels sur la question de savoir comment donner sens à son existence. La conséquence du paradoxe des séries est que je ne pourrai pas donner un sens à mon histoire personnelle si j'attends pour cela d'avoir trouvé ma ou mes fins principales, mon but dans l'existence. Autrement dit, les séries télévisées permettent de vivre une expérience sceptique du "paradoxe des séries", qui est finalement une forme télévisuelle de l'expérience 
sceptique de la contingence de l'existence ${ }^{34}$. Cependant, ce paradoxe des séries ouvre aussi la possibilité de reconnaître, avec Kate dans l'épisode S6E17, que "rien n'est irréversible ", c'est-à-dire que les séries peuvent nous permettre de prendre conscience de ce qu'il nous est toujours possible de changer et d'atteindre un meilleur état du moi. Roz Kaveney a ainsi bien mis en évidence l'importance de cette idée du changement personnel dans une série comme Buffy the Vampire Slayer (Joss Whedon, 1997-2003), où se multiplient les figures de shadow doubles, c'est-à-dire ces personnes que vous auriez pu être dans d'autres circonstances : on pense au personnage de Cordelia, la pom-pom girl, qui est ce que Buffy était elle-même avant d'avoir ses pouvoirs, ou bien à Faith, qui est une tueuse de vampires sans famille, ni amis, ou encore à la relation d'Angel à Spike ${ }^{35}$.

Cette idée est très importante dans Lost. Dès l'épisode S1E17, Locke dit que chacun commence une nouvelle vie sur l'île; Michael annonce à son fils qu'ils ont connu des contretemps, mais qu'ils vont tout reprendre à zéro et Sun demande à Jin s'ils pourraient recommencer à s'aimer. Ailleurs, Jacob justifie l'un des critères de sélection des candidats au statut de gardien de l'île par le fait qu'ils avaient tous intérêt à ce qu'une nouvelle chance leur soit donnée de devenir ceux qu'ils auraient dû être depuis toujours. Le " paradoxe des séries » vient donc confirmer le fait que les séries télévisées constituent un milieu d'expression privilégié pour ce que Stanley Cavell appelle le perfectionnisme moral ${ }^{36}$. Cependant, comment espérer donner sens à une série télévisée ou à sa vie si « rien n'est irréversible » et si l'on ne peut jamais être sûr de s'en représenter la fin? Le paradoxe sceptique ne nous conduit-il pas à l'alternative du " pur réalisme désenchanté » ou du « saut dans la foi »?

La série propose peut-être une autre solution, qui se rapprocherait de la définition kantienne de l'expérience esthétique. En effet, ce que la série nous fait vivre, ce dont nous faisons l'expérience en la suivant et en la comprenant sans connaître la fin, c'est que l'on peut donner un sens à une série ou à une existence en étant sensible à la forme finalisée qui semble s'y dessiner sans pour autant être en mesure de se former une représentation de sa fin. Cette expérience télévisuelle de la compréhension comme analogue de l'expérience esthétique au sens kantien est vécue en regardant Lost à deux moments au moins. Tout d'abord, dans ces scènes où des personnages sont contraints de sortir de leur rapport d'affairement et de consommation au monde pour entrer dans l'exercice spirituel de la contemplation ${ }^{37}$. Ensuite, à la fin, quand nous sommes déçus parce qu'en mettant un terme au « libre jeu de l'entendement et de l'imagination ${ }^{38}$ ", la série met aussi un terme au plaisir que nous y avons pris et nous renvoie à notre propre perte dans l'existence, celle de la perte du temps, de la perte d'une compagnie, celle qui nous oblige à trouver de nouvelles manières de renouer le contact avec le monde et les autres et à affronter la question de la forme de notre propre vie.

Cette hypothèse serait à relier au projet cavellien d'une « esthétique de l'ordinaire » et à l'idée que les œuvres d'art «veulent dire quelque chose pour nous, pas seulement à la manière d'énoncés, mais à la manière de personnes ${ }^{39}$. " La série propose, en effet, au spectateur une sortie du scepticisme et recommande une manière de surmonter l'expérience de la perte, dans une scène qui retrouve la façon dont des philosophes américains comme Emerson ou Thoreau ont cherché à imaginer la domestication du scepticisme ou encore l'habitation du monde, selon Stanley Cavell :«Pour Emerson et Thoreau, il faut apprendre à s'asseoir chez soi, à rester assis dans quelque lieu attirant dans les bois, comme pour se marier avec le monde, avant, si cela doit jamais être le cas, de prendre sur soi le fardeau des autres ${ }^{40} »$. Dans cette scène, Locke apprend à Boone à 
surmonter son désir fusionnel, mais destructeur pour Shannon et à accepter la réalité indépassable de leur séparation, ou mieux de leur être-séparé. Il en va de Boone comme de Locke, de Widmore ou du Projet Dharma, lorsqu'ils sont pris par un désir de connaissance exclusive des secrets de l'îl $e^{41}$ ou bien d'Othello quand il désire posséder Desdémone de corps et d'esprit : le désir de connaissance intime et exclusive du monde est mortel ; plus on cherche à s'assurer de la présence du monde et de l'autre, plus l'objet désiré s'éloigne et plus le scepticisme grandit.

Plutôt que de présupposer qu'il doit être possible de saisir et connaître les fins des personnages et du récit sans quoi rien n'aurait de sens, ce que nous avons à faire, à l'instar de Boone dans cette scène, c'est reconnaître le caractère séparé des personnages et du monde projeté en admettant que nous devons choisir de nous définir par nos réactions et notre compréhension, sans être certains que nous ne faisons pas que projeter des fins qui ne s'y trouvent pas. Autrement dit, s'il y a là une vérité du scepticisme, il y a aussi une erreur qui tient à ce que la présence au monde qui est cherchée n'est pas une présence qui pourrait être trouvée par la connaissance. Pour retrouver cette présence au monde perdue, il faut au contraire apprendre à nous passer de connaissance, c'est-à-dire renoncer à savoir que le monde existe et simplement l'« accepter ${ }^{42}$ » comme étant séparé de nous, renoncer à savoir que les autres existent pour mieux nous efforcer de " reconnaître ${ }^{43}$ » leur présence en nous révélant à eux, convaincus que « [c]'est la chose que nous ne savons pas qui peut nous sauver ${ }^{44}$."

L'expression récurrente dans la série pour signifier à la fois qu'il faut accepter d'avoir à prendre des décisions incertaines dans l'urgence et que c'est " la chose que nous ne savons pas qui peut nous sauver » est celle du «lâcher-prise » (« let go »). Elle correspond au procédé cinématographique qui consiste en un travelling avant, suivi d'un recadrage sur le visage en gros plan d'un personnage, qui permet d'introduire le flashback. Cette technique est classique et d'une compréhension très intuitive: la convention hollywoodienne veut que ce type de plan, appelé plan de réaction, constitue un moment de révélation de l'intériorité du personnage. Mais ce procédé a aussi un sens plus général puisqu'il exprime l'une des révélations que la série peut offrir à ses spectateurs, la série en nous montrant des personnages contraints par les circonstances d'interrompre le cours pressé de leur vie pour revenir en pensée sur leur vécu ${ }^{45}$.

\section{3) Surmonter le traumatisme par l'image : Lost ou l'expérience de la perte}

Dans son ouvrage Comment Hitchcock m'a guéri ${ }^{46}$, Serge Tisseron fait référence à l'analyse du phénomène psychologique du «clivage " proposée par l'un des premiers disciples de Freud, Sándor Ferenczi. Pour Ferenczi, un traumatisme grave peut induire une fracture de la personnalité en deux parties, «l'une, sensible, qui est brutalement détruite, et une autre qui sait tout, mais ne ressent rien ${ }^{47} »$. En effet, ce clivage permet à la victime de supporter le traumatisme, qui peut ainsi être « vécu » sans être " éprouvé ». Cependant, l'être clivé est désormais condamné à adopter une perspective d'observateur impartial sur son existence, plutôt que de participant que les événements émeuvent dans la mesure où ils le concernent au premier chef, ce qui fait que l'on peut dire de lui qu'il se trouve dans un état de vie apparente. On sait que chacun des candidats a connu un traumatisme et que c'est l'une des raisons pour lesquelles Jacob les a choisis. Or, les solutions prônées par Freud, se souvenir, ou par Ferenczi, éprouver enfin la catastrophe originelle, 
décrivent toutes deux des étapes importantes de la trajectoire des personnages de la série.

Cependant, Serge Tisseron prône une autre solution que celles de Freud ou de Ferenczi pour surmonter ce clivage. Cette solution passe par la métaphore, c'est-à-dire la recherche de mots, d'images ou de gestes qui permettraient à ceux qui souffrent de traumatisme de se donner des représentations personnelles du drame "suffisamment proches pour qu'ils se familiarisent avec lui, et en même temps assez éloignées pour ne mobiliser qu'une partie seulement des émotions qui y ont été éprouvées, ce qui permet de les cadrer et de les contenir facilement ${ }^{48}$."

Ce pouvoir de la métaphore rejoint la définition proposée par Jean-Pierre Esquenazi de la paraphrase comme ce qui se produit « quand l'appropriation du récit par un destinataire [le] conduit à comprendre l'univers fictionnel comme [...] exemplifiant [...] une part du monde rée ${ }^{49}$ ». Si ce rapprochement est correct, alors Lost paraphrase cette partie de nos existences qui relève du phénomène du clivage et qui fait que nous avons parfois l'impression de ne pas vivre les existences qu'il nous est donné de mener dans ce monde, d'être là sans être présents, bref de passer à côté de nos expériences. En effet, si nous n'avons pas tous eu le malheur de subir un traumatisme sévère au cours de nos vies, il est certain que le simple fait de vivre et de vieillir comporte une dimension suffisamment traumatisante pour que les phénomènes décrits par Ferenczi nous semblent étrangement familiers. L'une de ces expériences nécessaires, pour qui a la chance de vivre assez longtemps pour atteindre l'âge adulte, est celle de la perte : le passage de la jeunesse à l'âge adulte est en effet le passage de l'âge où tout est encore possible à celui où le sentiment de perte nous rappelle la réalité de ce que nous n'avons pas encore perdu.

Dans le chapitre 15 de La Projection du monde qui constitue une « digression sur un aspect de la peinture moderniste ", c'est-à-dire sur les lignes coulées de Jackson Pollock, les bandes de Morris Louis, les chevrons de Kenneth Noland ou les formes en Z de Frank Stella, Cavell a attiré notre attention sur une partie de «la signification du fait nouveau de la série, ou du fait qu'un nouveau moyen d'expression instaure, et est instauré par, une série $^{50}$ ", à savoir que si la peine et le baume de l'adolescence sont en effet l'idée de l'infini des possibles, « le seul profit du passage à l'âge adulte, la seule justice qu'il y a à renoncer à ce monde du possible, est la réception de la réalité (actuality) - la peine et le baume de la vérité du seul monde : qu'il existe, et moi dedans ${ }^{51}$. »Si Lost nous apprend à ne pas vieillir trop vite en ne cherchant pas dans notre âge et notre situation socio-professionnelle un prétexte pour ne plus chercher à changer et à devenir meilleurs, elle nous apprend aussi à accepter notre âge et à reconnaître que c'est l'expérience même de la perte qui donne son prix à l'existence qu'il nous est donné de vivre encore un peu en ce monde. En d'autres termes, renouer avec notre expérience, retrouver nos émotions supposera paradoxalement d'accepter de « lâcher prise ", en reconnaissant que c'est le désir de tout conserver, de contrôler entièrement sa vie, de ne rien perdre de ses souvenirs et de ses relations qui nous détruit et nous fait souffrir. C'est incontestablement là l'une des leçons de la fin de Lost qui permet ainsi aux spectateurs de se donner des représentations personnelles du drame « suffisamment proches pour qu'ils se familiarisent avec lui, et en même temps assez éloignées pour ne mobiliser qu'une partie seulement des émotions qui y ont été éprouvées ${ }^{52}$ ", c'est-à-dire de surmonter le traumatisme de la perte par l'image.

34 Cette leçon est aussi présente dans un film dont on ne saurait exagérer l'importance pour Lost, à savoir L'Échelle de Jacob (réal. Adrian Lyne, 1990), où, dans le passage central, l'ami de Jacob prétend citer Maître Eckhart : 
La seule chose qui brûle en enfer, c'est la part de toi qui s'accroche à la vie. Tes souvenirs, tes sentiments. Tout ça brûle. Mais pas pour te punir, dit-il... pour libérer ton âme. D'après sa théorie, si tu as peur de mourir... si tu te cramponnes... tu vois des démons t'arracher à la vie. Mais si tu as fait la paix en toi ces démons se muent en anges qui te libèrent de la terre ${ }^{53}$.

Figure 7 : Lost S1E1

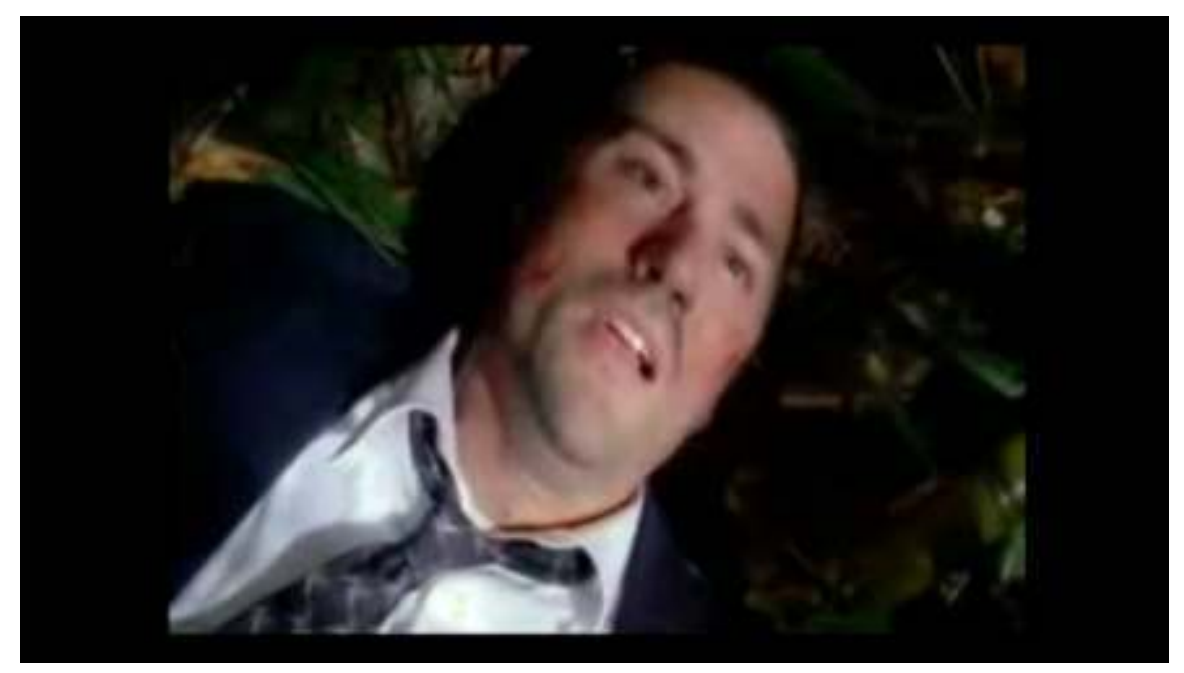

Figure 8 : L'échelle de Jacob

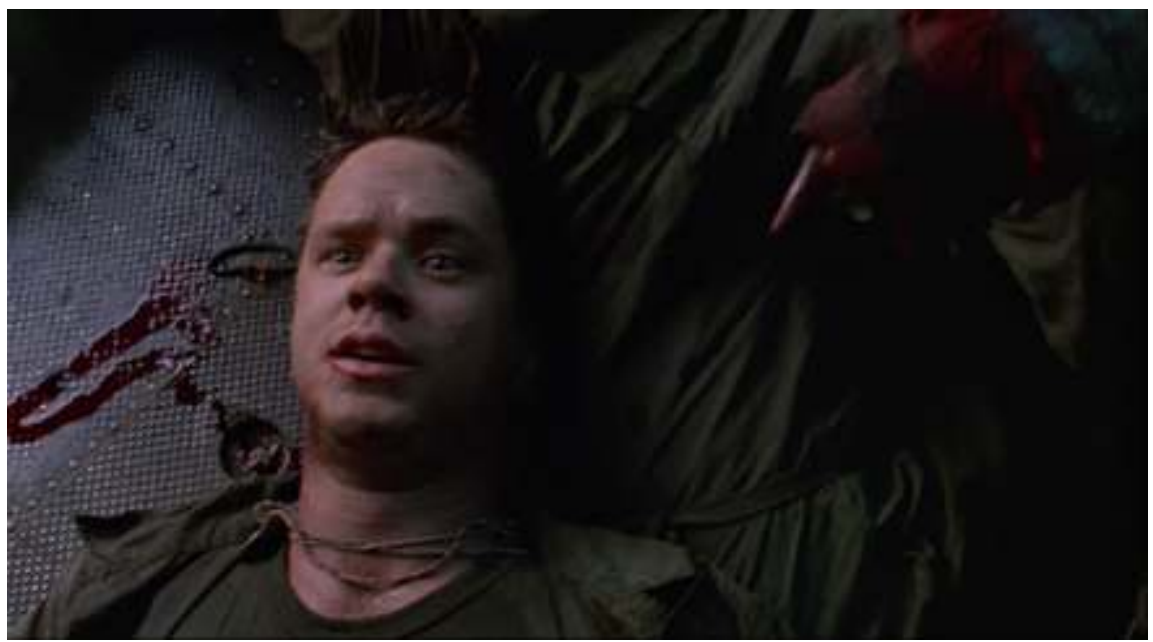


Figure 9 : Lost S6E18

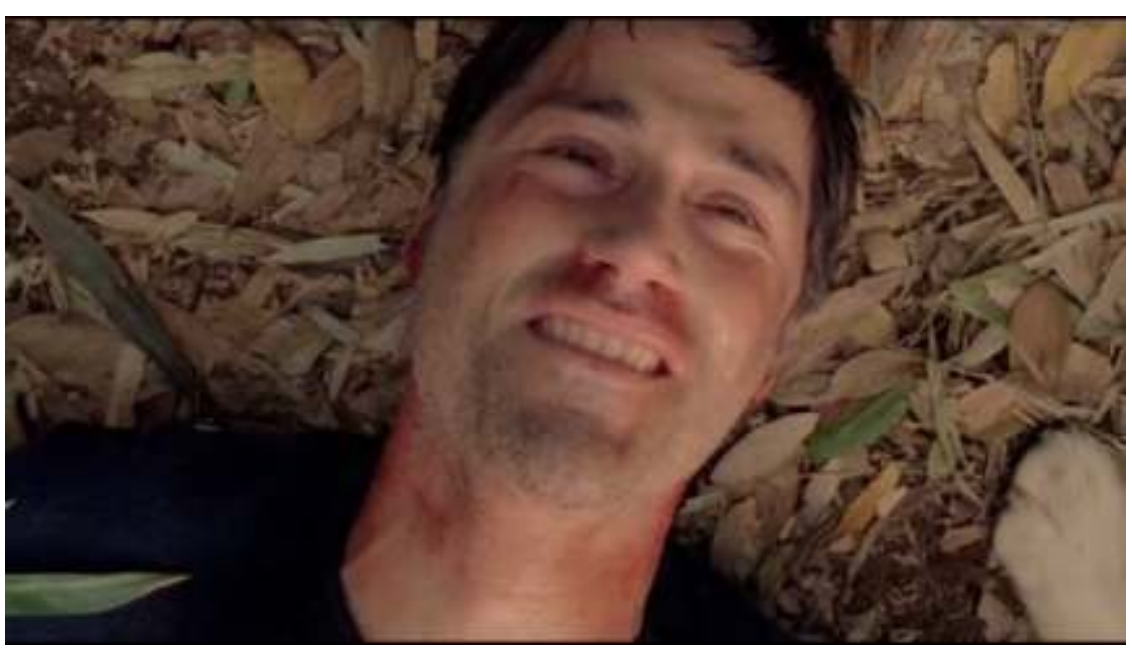

\section{Conclusion}

Le scandale causé par la fin de la série Lost soulève donc bien le problème de la clôture narrative, c'est-à-dire la question de savoir ce qui peut provoquer, ou manquer à provoquer, l'impression de finalité que l'on s'attend souvent à ressentir devant la majorité des œuvres audiovisuelles. Au modèle érotético-naturaliste de Noël Carroll, nous avons vu pourquoi il faut préférer le modèle téléologico-finaliste qui soutient que le sentiment de finalité tend à se produire quand l'œuvre fournit les éléments suffisants pour reconstruire les relations causales entre agents et patients naturels et les raisonnements pratiques des personnages principaux.

Cependant, ce modèle semble présupposer l'existence d'une hiérarchie des fins qui ne résiste pas au scepticisme moderne, ce qui est d'ailleurs pensé par Lost, une pensée de la série qui suppose, pour être comprise, de changer d'approche philosophique, c'est-à-dire non plus de philosopher sur les séries télévisées en adoptant une position de surplomb (qui correspond à ce qu'Arthur Danto nous a appris à condamner comme l'assujettissement de l'art par la philosophie ${ }^{54}$ ), mais de philosopher avec ou, mieux, d'après les séries télévisées, c'est-à-dire en prenant au sérieux les pensées qui s'y expriment. Lost est, en effet, une série qui aborde explicitement le problème sceptique d'avoir à donner du sens à l'existence en l'absence de toute nécessité téléologique, absence soulignée par l'une des caractéristiques du médium télésériel, à savoir qu'il est toujours possible de poursuivre une série télévisée autrement et de modifier ainsi rétrospectivement le sens des événements passés qui la constituent, ce que l'on peut appeler « le paradoxe des séries ». Mais ce paradoxe n'est que la forme télévisuelle d'une expérience sceptique ordinaire, qui n'est donc pas réservée seulement aux personnages, celle de la contingence de l'existence, qu'éprouve celui qui prend conscience de l'absence de finalité absolue. Susceptible de provoquer l'angoisse, cette incertitude quant au sens à donner à l'existence est aussi une chance, celle d'atteindre un meilleur état du moi, à condition de savoir imiter les personnages de Lost quand ils quittent leur rapport de consommation du monde, pour se rapporter à lui sur le mode d'une contemplation qui les rend sensibles à sa forme finalisée sans qu'ils puissent s'en représenter la fin. Ce rapport esthétique à l'ordinaire du monde n'est plus un rapport de connaissance, mais de 
reconnaissance, n'est plus une affaire de savoir, mais d'accepter d'être séparé du monde et des autres, une séparation qui s'éprouve dans l'expérience de la perte, ce qui est une affaire de savoir « lâcher prise ».

Loin d'être une insuffisance des scénaristes, l'absence de résolution satisfaisante à la fin de la série est donc plutôt une déclaration sur le rapport que nous devons entretenir avec les séries télévisées et nos existences. En effet, quand nous sommes déçus par la fin de la série, c'est non seulement parce qu'en nous faussant compagnie, la série met un terme au plaisir que nous y avons pris, mais surtout parce qu'elle nous renvoie à nos propres pertes et à la nécessité d'avoir à trouver de nouvelles manières de renouer le contact avec le monde et les autres, c'est-à-dire d'avoir à réinventer la forme de notre propre vie. La série dit ainsi que le juste rapport que nous devons adopter vis-à-vis d'elle est un rapport philosophique si, comme le souligne Wittgenstein, l'expérience philosophique caractéristique a la forme d'un "je ne m'y retrouve plus", c'est-à-dire d'un «je suis perdu », au sens où je perçois la forme d'une finalité sans pouvoir me représenter la fin.

\section{BIBLIOGRAPHIE}

ARISTOTE, Métaphysique, trad. J. Tricot, Paris, Vrin, 1991.

AUERBACH David, « The Cosmology of Serialized Television », The American Reader, juin 2013, http://theamericanreader.com/the-cosmology-of-serialized-television (consulté le 25 mai 2016).

CARROLL Noël, « Toward a Theory of Film Suspense », Persistence of Vision. The Journal of the Film Faculty of the City University of New York, $\mathrm{n}^{\circ}$ 81, 1984, p. 65-89 ; repr. in Theorizing the Moving Picture, New York, Cambridge University Press, 1996, p. 94-117.

__-. «The Power of the Movies », Daedalus, vol. 1, n 14, 4, automne 1985, p. 79-104; repr. in Theorizing the moving picture, p. 78-93.

_-_. « Narrative closure », Philosophical Studies: An International Journal for Philosophy in the Analytic Tradition, vol. 135, n $\mathrm{n}^{\circ}$, août 2007, p. 1-15 ; repr. in The Philosophy of Moving Pictures, Malden, MA, Blackwell, 2008, chap. 5, section 2, « Cinematic Narration », p. 133-46;

__-_. « Narrative closure », ed. Paisley Livingston et Carl Plantinga, The Routledge Companion to Philosophy and Film, Londres, Routledge, 2009, chap. 19, p. 207-216 ;

_-_. « Narrative closure ", Art in three dimensions, chap. 17, New York, Oxford University Press, 2010, p. 355-72.

Stanley Cavell, Dire et Vouloir Dire, Paris, Cerf, 2009 [1969].

_-_. La Projection du monde, Paris, Belin, 1999 [1979].

_-_. « Descartes, Emerson, Poe » [1985], trad. fr. Élise Domenach, Éthique, littérature, vie humaine, Sandra Laugier (dir.), Paris, Presses universitaires de France, "Éthique et philosophie morale », 2006, p. 211-244. 
CLEMOT Hugo, « Une lecture des films d'horreur épidémique », Tracés, « Contagions », coordonné par Florent Coste, Aurélien Robert et Adrien Minard, n²1, 2011/2, p. 167-184, http:// traces.revues.org/5209 (consulté le 25 mai 2016).

_-_. La Philosophie d'après le cinéma. Une lecture de La Projection du monde de Stanley Cavell, Rennes, Presses universitaires de Rennes, 2014.

DANTO Arthur, L'assujettissement philosophique de l'art, Paris, Seuil, 1993 [1986].

DAVIES Richard, « Lost in Lost's Time », The Ultimate Lost and Philosophy: Think Together, Die Alone, ed. Sharon Kaye, Hoboken, New Jersey, John Wiley \& Sons, Inc., 2011, p. 9-31.

ESQUENAZI Jean-Pierre, La vérité de la fiction, Paris, Lavoisier, 2009.

FAUTRIER Pascale, « Le cinéma de Sartre », Fabula-LhT, n 2, « Ce que le cinéma fait à la littérature (et réciproquement) », décembre 2006, http://www.fabula.org/lht/2/fautrier.html (consulté le 25 mai 2016).

FERENCZI Sándor, « Analyse d'enfants avec des adultes », Psychanalyse, t. 4, Paris, Payot, 1982 [1931].

HATCHUEL Sarah, Lost : Fiction vitale, Paris, Presses universitaires de France, 2013.

KANT Emmanuel, Critique de la faculté de juger, trad. fr. A. Renaut, Paris, GF Flammarion, 2000.

KAVENEY Roz, Reading the Vampire Slayer: The Complete, Unofficial Guide to Buffy and Angel, New York: I.B. Tauris, 2004.

KRIPKE Saül, Règles et langage privé. Introduction au paradoxe de Wittgenstein, trad. T. Marchaise, Paris, Seuil, 1996 [1982].

LANG Michelle A., « Lost: poststructural metanarrative or postmodern Bildungsroman? » [2007], LOST Thought. Leading Thinkers Discuss LOST, ed. Pearson Moore, Vancouver, Inukshuk Press, 2012, p. 307-312.

LAUGIER Sandra, «Vertus ordinaires des cultures populaires », Critique, $\mathrm{n}^{\circ} 776-777$, janvierfévrier 2012, p. 48-61.

MOREAU Denis, Dans le milieu d'une forêt. Essai sur Descartes et le sens de la vie, Paris, Bayard, 2012

POMARES Claire, « How I Met Your Mother, pire fin de série de tous les temps ? ", Les Inrocks, $1^{\text {er }}$ avril 2014, http://goo.gl/Rxtqvv (consulté le 25 mai 2016).

POUDOVKINE Vsevolod I., Film Technique in Film Technique and Film Acting, New York, Grove Press, Inc., 1958.

THIELLEMENT Pacome, Les mêmes yeux que Lost, Paris, Léo Scheer, 2011.

TISSERON Serge, Comment Hitchcock m'a guéri. Que cherchons-nous dans les images ?, Paris, Pluriel, 2014 [2003].

VELLEMAN J. David, « Narrative explanation », The Philosophical Review, vol. 112, n 1, janvier 2003, p. 1-25.

WILSON George M. , Narration in Light, Baltimore, John Hopkins University Press, 1986.

WITTGENSTEIN Ludwig, Recherches philosophiques, trad. fr. Françoise Dastur et al., Paris, Gallimard, 2004. 


\section{NOTES}

1. Voir l'article des Inrocks à propos de la fin de How I Met Your Mother (Carter Beys, Craig Thomas, 2005-2014) qui sous-titre « Le Lost des sitcoms » ; Claire Pomarès, « How I Met Your Mother, pire fin de série de tous les temps? ", Les Inrocks, $1^{\mathrm{er}}$ avril 2014, http://goo.gl/Rxtqvv (consulté le 25 mai 2016).

2. Noël Carroll, «Toward a Theory of Film Suspense », Persistence of Vision. The Journal of the Film Faculty of the City University of New York, $n^{\circ} 81,1984$, p. 65-89; repr. in Theorizing the Moving Picture, New York, Cambridge University Press, 1996, p. 94-117 ; "The Power of the Movies ", Daedalus, vol. 1, nº 14, 4, automne 1985, p. 79-104; repr. in Theorizing the moving picture, p. 78-93 ; "Narrative closure ", Philosophical Studies: An International Journal for Philosophy in the Analytic Tradition, vol.135, $\mathrm{n}^{\circ}$ 1, août 2007, p.1-15; The Philosophy of Moving Pictures, Malden, MA, Blackwell, 2008, chap. 5, section 2, "Cinematic Narration», p. 133-46; "Narrative closure ", Paisley Livingston et Carl Plantinga (éd.), The Routledge Companion to Philosophy and Film, Londres, Routledge, 2009, chap. 19, p. 207-216 ; Art in three dimensions, chap. 17, "Narrative closure », New York, Oxford University Press, 2010, p. 355-72.

3. Carroll, « Narrative closure », Philosophical Studies, p. 1.

4. Carroll, « Narrative closure », Routledge Companion, p. 207 ; Art in three dimensions, p. 367.

5. Carroll, «On Narrative Connection», Beyond Aesthetics, Cambridge, Cambridge University Press, 2001, p. 127 ; Art in three dimensions, p. 367.

6. Vsevolod I. Poudovkine, Film Technique in Film Technique and Film Acting, New York, Grove Press, 1958, p. 73; 77.

7. Carroll, « Toward a Theory of Film Suspense », Theorizing the Moving Picture, p. 96.

8. Carroll, « Narrative closure », Routledge Companion, p. 213 ; Art in three dimensions, p. 366.

9. Voir, par exemple, Richard Davies, «Lost in Lost's Time ", in The Ultimate Lost and Philosophy: Think Together, Die Alone, ed. Sharon Kaye, Hoboken, New Jersey, John Wiley \& Sons, Inc., 2011, p. 9-31.

10. Carroll, « Narrative closure », The Routledge Companion, p. 213 ; Art in three dimensions, p. 366. Le cas du tollé provoqué par le cliffhanger du dernier épisode de la saison 6 de The Walking Dead (F. Darabont et R. Kirkman, AMC, 2010-) en constitue un bon exemple récent, les auteurs s'étant même excusés auprès des fans de n'avoir pas révélé le nom de la victime de Negan, contrairement à ce que les teasers diffusés par la chaîne AMC promettaient avant même le début de la diffusion de la saison. Pour un échantillon des réactions glanées sur Internet, voir https:// goo.gl/fwMemF (consulté le 26 mai 2016).

11. "Okay, that thing in the woods, maybe it's a monster, maybe it's a pissed-off giraffe, I don't know. The fact that no one is even looking for us - yeah, that's weird. But I just go along with it... 'cause I'm along for the ride - good old fun-time Hurley. Well, guess what - now, I want some freakin' answers! » «OK, cette chose, dans les bois, c'est peut-être un monstre, ou une girafe en colère. J'en sais rien ! Le fait que personne ne nous cherche, oui, c'est bizarre. Mais je m'en arrange. Je suis partant pour l'aventure. Ce bon vieux clown d'Hurley ! Eh bien, y a du nouveau ! Maintenant, je veux des foutues réponses!»

12. J. David Velleman, "Narrative explanation", The Philosophical Review, vol. $112, \mathrm{n}^{\circ} 1$, janvier 2003, p. 1-25.

13. " Each Active's brain was programed to release a sedative the moment they felt closure ».

14. George M. Wilson écrit en ce sens que «les questions importantes d'un point de vue dramatique qu'un film de fiction peut soulever à propos de ses personnages sont en effet illimitées. » Voir George M. Wilson, Narration in Light, Baltimore, John Hopkins University Press, 1986, p. 50. 
15. Cet exemple de Carroll est problématique. On peut avoir regardé le film en le comprenant sans avoir été agacé par le fait qu'il présente essentiellement le point de vue du grand frère.

16. Voir le commentaire de David Auerbach dans " The Cosmology of Serialized Television ", The American Reader, juin 2013, http://theamericanreader.com/the-cosmology-of-serializedtelevision/ (consulté le 25 mai 2016).

17. Dans une sorte d'illustration visuelle frappante du pacte de soumission au Léviathan de Thomas Hobbes.

18. Aristote, Métaphysique, VII, 7 1032b7-10, trad. J. Tricot, Paris, Vrin, 1991, p. 261.

19. Carroll, « Toward a Theory of Film Suspense », p. 98.

20. Carroll, The Philosophy of Motion Pictures, p. 133.

21. C'est à peu près la conclusion de Davies dans son article sur les problèmes conceptuels posés par l'idée de voyage dans le temps : tant que les scénaristes font leur travail, qui consiste à nous aider à ne pas penser à ces contradictions en nous suggérant de chercher plutôt des réponses à des questions relatives à ce que vivent les personnages, nous pouvons être satisfaits et trouver du plaisir à suivre la série. Richard Davies, «Lost in Lost's Time », p. 29-30.

22. Voir Pacome Thiellement, Les mêmes yeux que Lost, Paris, Léo Scheer, 2011.

23. Pacome Thiellement à propos de Lost au Forum des images, le 20 avril 2012, dans le cadre du festival Séries mania : http://goo.gl/I3rSbW (consulté le 25 mai 2016).

24. "After everything that I have been put through you owe me an answer. "

25. Sur l'importance de ce texte de Descartes, voir Denis Moreau, Dans le milieu d'une forêt. Essai sur Descartes et le sens de la vie, Paris, Bayard, 2012.

26. Voir Hugo Clémot, "Une lecture des films d'horreur épidémique ", Tracés, "Contagions ", coordonné par Florent Coste, Aurélien Robert et Adrien Minard, n²1, 2011/2, p. 167-184, http:// traces.revues.org/5209 (consulté le 25 mai 2016).

27. "Welcome to the wonderful world of not knowing what the hell's going on. "

28. Les nombreuses expériences d'hallucination ou de rêve fonctionnent comme des reprises de l'argument sceptique du rêve cartésien. Voir Sarah Hatchuel, Lost: Fiction vitale, Paris, Presses universitaires de France, 2013, p. 52.

29. Hatchuel, p. 30.

30. Saül Kripke, Règles et langage privé. Introduction au paradoxe de Wittgenstein, trad. T. Marchaise, Paris, Seuil, 1996 [1982].

31. Ibid., p. 74.

32. Ludwig Wittgenstein, Recherches philosophiques, § 201, trad. fr. Françoise Dastur et al., Paris, Galllimard, 2004, p. 126.

33. Voir l'épisode « Numbers» (S01E18).

34. Il y aurait à réfléchir au curieux retournement que le paradoxe des séries pourrait produire sur la question des relations entre le cinéma et l'existentialisme. En effet, dans un article intitulé «Le cinéma de Sartre », Pascale Fautrier nous apprend que «c'est dans [un] texte de 1931 sur le cinéma qu'est formulée pour la première fois cette opposition majeure dans la pensée sartrienne entre nécessité de l'art et contingence de l'existence; et c'est à propos du cinéma qu'elle est formulée. » Elle cite les propos éclairants de Sartre dans les entretiens filmés de 1972 qui donneront le film Sartre par lui-même (réal. Alexandre Astruc, Michel Contat et Guy Séligmann, 1976): "Je sais que l'idée de contingence est venue de la comparaison qui s'est établie spontanément chez moi entre le paysage dans un film et le paysage dans la réalité. Le paysage d'un film, le metteur en scène s'est arrangé pour qu'il ait une certaine unité et un rapport précis avec les sentiments des personnages. Tandis que le paysage de la réalité n'a pas d'unité. Il a une unité de hasard et ça m'avait beaucoup frappé. Et ce qui m'avait beaucoup frappé aussi, c'est que les objets dans un film avaient un rôle précis à tenir, un rôle lié au personnage, alors que dans la réalité les objets existent au hasard ", cité dans Jean-Paul Sartre, Euvres romanesques, Gallimard, Bibliothèque de la Pléiade, p. 1698. Voir Pascale Fautrier, « Le cinéma de Sartre », Fabula-LhT, n², 
"Ce que le cinéma fait à la littérature (et réciproquement) ", décembre 2006, http:// www.fabula.org/lht/2/fautrier.html (consulté le 25 mai 2016).

35. Roz Kaveney, "She saved the world. A lot. An introduction to the themes and structures of Buffy and Angel ", Reading the Vampire Slayer: The Complete, Unofficial Guide to Buffy and Angel, New York, I.B. Tauris, 2004, p. 10.

36. Voir Sandra Laugier, «Vertus ordinaires des cultures populaires », Critique, $\mathrm{n}^{\circ} 776-777$, janvier-février 2012, p. 48-61.

37. Voir Michelle A. Lang, «Lost: poststructural metanarrative or postmodern Bildungsroman?» [2007], LOST Thought. Leading Thinkers Discuss LOST, ed. Pearson Moore, Vancouver, Inukshuk Press, 2012, p. 308 : «Suddenly torn from their usual lives, they have plenty of time, and stimuli, on the island to provoke a meditation on who they really are and how they got to this point, on core beliefs that no longer seem valid, and on maps of the world inherited from family systems that now need to be redrawn. " «Subitement arrachés à leurs vies quotidiennes, ils ont sur l'île beaucoup de temps, et d'occasions, pour méditer sur ce qu'ils sont réellement et comment ils en sont arrivés là, sur leurs intimes convictions qui ne semblent plus adéquates, et sur les cartes du monde dont ils ont hérité en famille et qui doivent être redessinées. »

38. Emmanuel Kant, Critique de la faculté de juger, "Analytique du beau », §9, trad. fr. A. Renaut, Paris, GF Flammarion, 2000, p. 196.

39. Stanley Cavell, « De la décomposition de la musique », Dire et Vouloir Dire (DVD, ensuite), Paris, Cerf, 2009 [1969], p. 325.

40. Cavell, «Descartes, Emerson, Poe» (1985), trad. fr. Élise Domenach, Éthique, littérature, vie humaine, Sandra Laugier (dir.), Paris, Presses universitaires de France, «Éthique et philosophie morale », 2006, p. 243.

41. Voir Hatchuel, p. 90.

42. Cavell, « L'évitement de l'amour », DVD, p. 483.

43. Cavell, loc. cit. Voir surtout « Connaître et reconnaître », DVD, p. 377-412.

44. Cavell, « L'évitement de l'amour », DVD, p. 485.

45. Voir Hatchuel, p. 74-75: «À travers ces flashs, les rescapés font leur propre analyse émotionnelle et se libèrent peu à peu de leur prison personnelle [...] ce qui leur permettra d'évoluer moralement. »

46. Serge Tisseron, Comment Hitchcock m'a guéri. Que cherchons-nous dans les images?, Paris, Pluriel, 2014 [2003], p. 79.

47. Sándor Ferenczi, « Analyse d'enfants avec des adultes », Psychanalyse, t. 4, Paris, Payot, 1982 [1931], p. 106.

48. Tisseron, p. 84.

49. Jean-Pierre Esquenazi, La vérité de la fiction, Paris, Lavoisier, 2009, p. 155.

50. Cavell, La Projection du monde, Paris, Belin, 1999 [1979], p. 159. Voir Hugo Clémot, La Philosophie d'après le cinéma. Une lecture de La Projection du monde de Stanley Cavell, Rennes, Presses universitaires de Rennes, 2014, p. 163-176.

51. Cavell, La Projection du monde, p. 161 [trad. modifiée par l'auteur].

52. Tisseron, Comment Hitchcock m'a guéri, p. 84.

53. "The only thing that burns in Hell is the part of you that won't let go of life, your memories, your attachments. They burn them all away. But they're not punishing you, he said. They're freeing your soul. So, if you're frightened of dying and... you're holding on, you'll see devils tearing your life away. But if you've made your peace, then the devils are really angels, freeing you from the earth. "

54. Arthur Danto, L'assujettissement philosophique de l'art, Paris, Seuil, 1993 [1986]. 


\section{RÉSUMÉS}

La question de savoir pourquoi tant de spectateurs furent si déçus par la fin de Lost pose le problème de la clôture narrative. Pour le résoudre, Noël Carroll a proposé un modèle « érotétique ", qui soutient que l'impression de finalité que l'on ressent à la fin de la plupart des films et des épisodes est provoquée par le fait que les questions que se pose le spectateur trouvent finalement une réponse. Cette théorie expliquerait l'enthousiasme suscité par une série qui accumule les mystères, mais aussi la déception finale de ne pas avoir reçu toutes les clés pour les comprendre. Si ce modèle érotétique présente incontestablement un intérêt au moins formel, on peut montrer, à partir de Lost, qu'il est néanmoins préférable de penser les choses selon un modèle téléologique où la subordination de certaines questions et réponses à d'autres renvoie en fait à un rapport entre moyens et fins. Cependant, on présuppose alors qu'il doit être possible de connaître les fins des personnages et du récit, sans quoi rien n'aurait de sens, alors que nous ne pouvons que reconnaitre le caractère séparé des personnages et du monde projeté, et admettre que nous sommes condamnés à comprendre sans jamais être certains que nous ne fassions pas que projeter des fins qui ne s'y trouvent pas. Or, les séries télévisées permettent de vivre une expérience sceptique, créée par ce que l'on peut appeler le " paradoxe des séries ", c'est-à-dire l'idée qu'il est toujours possible de poursuivre une série autrement et de modifier ainsi rétrospectivement le sens des nombres ou des événements passés qui la constituent, une vérité sceptique qui menace aussi l'histoire personnelle du spectateur, tout en lui offrant la possibilité de changer et d'atteindre un meilleur état du moi. Pour surmonter cette épreuve sceptique, la série nous apprend à contempler la forme d'une finalité qui semble se dessiner dans toute vie, sans qu'on puisse se former une représentation de sa fin, une leçon qui justifie que l'on puisse trouver la fin de Lost réussie.

That the viewers of the Lost TV series were so disappointed by its last episode has something to do with the philosophical problem of narrative closure. To resolve this conceptual difficulty, Noel Carroll has conceived an 'erotetic' model in which the impression of finality someone usually feels at the end of most films and TV episodes is due to the fact that the questions the viewers ask themselves in the course of following the plot finally get an answer. This theory thus seeks to explain not only the enthusiasm which the TV series succeeds in eliciting by intriguing viewers with accumulating mysteries, but also how the viewers feel let down in the end when they realize they have not been given all the keys to solve these mysteries. This erotetic model surely has at least a formal interest. One, however, has reasons to prefer, and reasons that can be found in Lost , a teleological model where the relations between questions and answers are better understood when we see them as a question of the relation between means and ends. Yet this teleological model presupposes that we can somehow know the goals of the characters and of the narrative. If that were not the case, nothing would make sense, indeed. Still we can only acknowledge our separateness from the characters and from the projected world and admit that we have no choice but to understand without being certain that we are not simply projecting goals that do not exist outside of our minds. TV series allow us to live a skeptical experience, which originates in what can be called the 'series-following paradox' which is that we could always choose to follow a numerical series or a TV series and to retrospectively modify the meaning of the numbers or past events which constitute it. This skeptical truth not only threatens the TV experience we undergo 
while watching our favorite shows, but it also affects our perception of the meaning of our own personal lives. It also gives us a chance to change and to reach a better self. In order to surmount this skeptical trial, the Lost TV series teaches us to contemplate the form of a finality which seems to design life, without being able to get a representation of its goal, a lesson which actually enables us to see the ending of Lost as a success.

\section{INDEX}

Mots-clés : Lost, clôture, narration, Carroll Noël, modèle érotétique, modèle téléologique, paradoxe des séries, expérience esthétique

Keywords : Lost, closure, Carroll Noël, erotetic model, teleological model, parodox of series, aesthetic experience

\section{AUTEUR}

\section{HUGO CLÉMOT}

Hugo Clémot est agrégé, docteur en philosophie et chargé de cours à l'Université de Tours. Spécialiste de philosophie du cinéma, associé au Centre de philosophie contemporaine de la Sorbonne (ISJPS), il a publié les ouvrages : Les Jeux philosophiques de la trilogie Matrix (Vrin, 2011), La Philosophie d'après le cinéma (PUR, 2014) et Enseigner la philosophie avec le cinéma (dir., Les Contemporains favoris, 2015). CEuvrant par ses articles et ses interventions à la diffusion des philosophies analytiques et post-analytiques du cinéma, il cherche aussi à contribuer à la création d'une philosophie des séries télévisées.

Hugo Clémot, laureate of the agrégation, holds a PhD in philosophy and teaches at the university of Tours. A specialist of the philosophy of cinema and a member of the "Centre de philosophie contemporaine de la Sorbonne", he is the author of the following books: Les Jeux philosophiques de la trilogie Matrix (Vrin, 2011), La Philosophie d'après le cinéma (PUR, 2014) and Enseigner la philosophie avec le cinéma (ed., Les Contemporains favoris, 2015). He aims at making philosophical (post)analyses of cinema more accessible and at creating a philosophy of TV series. 